\title{
Scorpion Sting Envenomation
}

\author{
Lakhhar B', Patil MM², Patil SV ${ }^{3}$
}

${ }^{1}$ Dr. Bhavana B Lakhkar, MBBS. MD, Professor, ${ }^{2} \mathrm{Dr}$ MM Patil, MBBS, MD, Associate Professor, ${ }^{3} \mathrm{Dr}$ SV Patil, MBBS, MD, Professor and Head of Department. All from the Department of Paediatrics, Dr. BM Patil Medical College, Vijaypur BLDE University Vijaypur, Karnataka, India.

Address for correspondence:

Dr. Bhavana B Lakhkar

E-mail: blakhkar@yahoo.co.in

\section{How to cite}

Lakhhar B, Patil MM, Patil SV. Scorpion Sting Envenomation. J Nepal Paediatr Soc 2016;36(3):284-287

\begin{abstract}
Scorpion sting envenomation is very common problem in rural India especially where the climate is hot and dry. Management techniques have improved tremendously over the time leading to reduced morbidity and mortality. Mortality is mainly when ignorant patients go to the traditional faith healers(Tantrics) and come late to the facility where better management is available. Indian red scorpion (Mesobhuthustamulus) is the main species in India. Autonomic storm caused by venom acting on sodium gated and other channels is the main cause of manifestations in a scorpion sting victim. Cardio-myopathy is the main cause for morbidity and mortality. Many gradingsystems have been suggested for categorizing and simplifying the treatment. The mainstay of treatment where systemic involvement is there are alpha-1 blocker Prazosin hydrochloride, Fluids and newly arrived purified anti-venom.Scorpion envenomation being a problem of rural remote areas and most patients being from lower socio-economic status, anti-venom use is limited. Hence the treatment of choice remains Prazosin hydrochloride. Risk factors, peculiarities of presentation and dose titration of Prazosin based on severity will be discussed as per personal experience and literature review.
\end{abstract}

Key words: Scorpion sting, Autonomic storm, Prazosin, antivenom.

\section{Introduction}

T here are around 1500 species of scorpion in the world and only 30 are poisonous. Venomatous scorpions are mainly from Buthidae family. Different species are found in different geographical area and have different manifestations ${ }^{1}$. Most affected countries in world are from Africa especially Tunisia and most affected country in Asia is India.

As different manifestation are observed in different geographical areas due to variation of scorpion species and their venoms,the management also differs. In India Mesobuthustamulus species (Indian red scorpion) is common, whereas Centruroidsis common in north and Central America. Indian red scorpion mainly affects heart but Centruroid species affects central nervous system. In Trinidad pancreatitis is common and in Iran tissue necrosis and hemolysis is seen $^{2}$.In spite of this variation in presentation, in any one geographical 
area typical sequence of manifestations are observed. Hence the sequential management will remain same in that area and it is possible to make a protocol for management ${ }^{3}$. This will simplify the treatment.

\section{Risk factors for Severityof envenomation}

\section{Factors in the victim:}

Age:Children show severe disease as amount of poison becomes more for their tiny weights ${ }^{1} \cdot \ln$ our experience age below 3 yrs had severe symptoms and recovery was delayed ${ }^{4}$.

Time interval between sting and treatment: Most important risk factor described by most authors ${ }^{2,5}$ and also observed by us $^{4,6}$. Mortality increases with increase in time.

Site of sting:Envenomation is more severe if sting is near head and trunk though extremities are more commonly involved.

Other factors in Victim:Apart from age discussed above, general health and weight(nutritional status) of patient are important.

Factors in scorpion:Type of species, size of scorpion and the sting, venom composition and depth of sting are important factors.

Drugs received:If patient has received steroids, antihistaminics or diuretics like Furosemide, the mortality and morbidity both increase. Many of our children receive steroids and diuretics before they arrive to the facility.

\section{The Venom}

Different species of scorpion produce venom of different composition.It is a complex protein witheither Alpha, beta or both chains. Venom of Indian red scorpion has alpha chain toxin and acts on voltage gated sodium channels. Other species' venoms can act of Potassium or calcium channels. The venom keeps channels, open and active leading to persistent depolarization and release of neurotransmitters from adrenal medulla and autonomic nerves. This leads to so called "autonomic storm"with typical clinical features ${ }^{5,6,7}$. Scorpion venom is more toxic than snake on weight to weight basis but total quantity inoculated is less ${ }^{2}$.

\section{Clinical features}

Local pain:Approximately $75 \%$ of scorpion sting victims have only local symptoms like severe pain and parasthesia due to serotonin in the venom. The pain may last for $12-16$ hours. There may be some swelling and inflammation. This is popularly called dry stings. A tap at the sting site may cause severe pain (Tap test). Many times when systemic symptoms are severe, local pain may not be felt by patient and pain appears when symptoms resolve. This is a useful sign of recovery 4 . In our experience in children below 2 yrs,sudden cry followed by autonomic storm is enough to start treating as scorpion sting.

Systemic symptoms:Systemic symptoms vary depending upon species of scorpion hence differ from area to area. Indian red scorpion has autonomic storm as main feature. Parasympathetic activity like profuse sweating, vomiting, salivation restlessness and hypotension are first to appear. This lasts for 30-40 minutes and is followed by sympathetic activity like tr emors,anxiety,tachycardia,tachypnea, hypertension and cold extremities due to vaso-constriction.This stage lasts for 6-12 $\mathrm{hrs}^{8,9}$.Patients are usually brought in this phase as first phase goes in travelling or with the tantriks. They are usually dehydrated due to fluid loss in first stage and may have hypotension due to hypovolemia. Hypertension might appear as dehydration is corrected.

Complications: The most common complication in Indian red scorpion is cardiac involvement. Tachycardia persists or progresses to bradycardia, patient may develop premature beats, arrhythmias, gallop rhythm or congestive cardiac failure and pulmonary edema. Pulmonary edema is the most common cause of deathin our patients and is common when treatment is delayed.

Neurological complications are rare in India but are common withCentruroid species found in southern America and androctonus species in Tunisia. Patients usually present with coma, convulsions, pupillary changes and cerebral edema. In India stroke like features may be seen and are due to disseminated intravascular coagulation.

Other organs also can get affected due to inflammatory mediators. Pancreatitis is very common in Trinidad ${ }^{2}$.

\section{Investigations}

Leukocytosis with poly-morphonuclear predominance develops within hours ${ }^{6}$.ESR may be raised.Hyperglycemia due to catcholamine surge and increased counterregulatory hormones is very common and was seen in $60-80 \%$ of our cases. Hyponatremia, 
hypocalcemia, hypokalemia and abnormal coagulation profile may be observed. Depending upon organ of involvement, cardiac enzymes, liver enzymes,serum amylaseand renal function test may be abnormal ${ }^{8,9}$. Cardiac enzymes are helpful in diagnosing, confirming and deciding the management ${ }^{6}$. We found CPK MB very useful.

X-ray Chest:May show classical batwing appearance of pulmonary edema. Some patients may show evidence of aspiration due to forced feeding by parents.

Electrocardiogram is a useful noninvasive test, available even in the rural settings. In India where cardiac complications are common with Mesobuthustamulus it is very useful.ST segment and T wave changes,prolong QT interval and arrhythmias can easily be diagnosed ${ }^{6}$.

Echo-cardiogram is useful and sensitive investigation in India where cardiac complications are common.Low ejection fractions, motion abnormalities of ventricular wall are commonly seen. In our experience normal $\mathrm{ECHO}$ at discharge is assuring.

\section{Management}

Many grading systems have been suggested ${ }^{10,11}$ to assess severity,for planning therapy and predicting mortality. These were specific for a species but in 2011 a consensus statement was made based on experience of 16 scientists who were representatives of endemic countries. This was suitable for all species ${ }^{11}$. The use of grading system is mainly to simplify the treatment. As for now following modalities of treatment are available.

1. Analgesics for local pain: most commonly used.

2. Alpha 1 blocker phosphodiesterase inhibitor Prazosin hydrochloride: The key drug

3. Supportive treatment: Fluids,ionotropes specially dobutamine,vasodilatorantihypertensives like Nifedepine, Nitroglycerine etc.

4. Scorpion antivenom

Scorpion sting is a problem of developing countries, where cost of treatment is always anissue and hence in most places prazosin and supportive treatment is the mainstay of therapy ${ }^{1,4,6}$.

Prazosin is called a physiological, symptomatic, and pathological antidote. It acts by blocking autonomic storm. The prescribed dosage is 30 microgram $/ \mathrm{kg}$ every three hourly till symptoms subside.Usually three to five doses are required. Mortality has decreased to less than
$1 \%$ after the use of Prazosin ${ }^{12}$. Prazosin is cheap, easily available,easy to administer (oral) and very effective, hence remains the key drug for management of scorpion sting in India.

Antivenin theoretically is the specific management of scorpion sting and now available in most countries. Purified anti-venom $\mathrm{F}(\mathrm{ab}) 2$ has been widely used now. During parasympathetic phase venom is free in blood, in this period antivenom is most effective and neutralizes free venom in 4-6 hours. This prevents sympathetic storm which follows. Only problem with anti-venom is, its cost and availability in rural areas. We could manage our patients without venom with minimal mortality, which was mainly due to delay in patient arrival.

In our institution we observed that in severe envenomation if patient receives higher dose (60$90 \mathrm{mcgm} / \mathrm{kg}$ ) of Prazosin,the response is dramatic and survival is better. There was no adverse effect of high dose. This was an observation when patients received additional doses accidentally due to shift from one hospital to other. This observation needs further studies to prove whether high Prazosin can be a cheaper alternative for antivenom ${ }^{4}$

Availability of grading system has made it possible to have protocols for scorpion sting management. Grade I(only pain) needs only analgesics, grade II (hypotensio $\mathrm{n}$,salivation,vomiting)should be treated with intravenous fluids first and when blood pressure is stabilized and patient is able to swallowPrazosin should be given. lonotropes (Dobutamine)should be used only if patient presents late with cardio-myopathy or pulmonary edema ${ }^{3}$.In our personal experience evenif hypotension and fluid loss was present only maintenance fluids and prazosin were found to be effective. There is always a risk of fluid overload due to involvement of heart so better to manage with only maintenance fluids.

\section{Prevention}

A step towards prevention is the need of hour. In the first place sting by scorpion should be avoided.Hiding places for scorpion inside the house are behind long stored, grain bags, boxes etc in storage areas, crevices in the walls or roofs, clothes and shoes not used for few days.Outside the house barks of trees, under surface of a stones, bushes, crevices in the fields ${ }^{1}$. People in rural areas often make children sleep in storerooms, walk in fields with bare feet,carry grain bags on their backs without checking. They should be trained for frequent cleaning of storage area and not to use it for human dwelling. Closed shoes should be used for field, not even 
sandals (8). Cloths and shoes kept for long time,should be shaken before use. Roofs if made of tiles, false roof will be protective. Crevices should be filled up regularly. Pesticide sprays like DDT, Pyrithrine also are useful.

In case of suspected bites people should be educated to go to medical facility rather than traditional healers.Prazosin tablets should be made available in all rural hospitals and medical stores.It also has been suggested to keep some tablets in commonly approachable places like outpatient department ${ }^{13}$ or other small shops in rural areas. In rural areas doctors carry Prazosin tablets with them wherever they go $^{14}$. Education campaign for preventive measures, awareness about availability and effectiveness of treatment should be conducted regularly in rural areas. Trials for vaccines are on the way.

\section{Conclusion}

In our personalexperience, after dealing with scorpion sting envenomation for so many years, wethink, it is a preventable, treatable problem in rural area. Age of patient, time interval between sting and seeking treatment, are two most important risk factors. Autonomic storm is the most common manifestation in India and Pulmonary edema secondary to heart damage is the most common cause of death. Prazosin hydrochloride, an alpha receptor blocker given orally is cheap and effective remedy. Given in time, it is a life saver.Anti-scorpion venom though useful, is available in selected areas and cost is a constraint. Prevention is possible by simple lifestyle changes for which people in rural setup should be educated.

\section{References}

1. BawaskarHS, Bawaskar PH. Scorpion sting: update. JAPI 2012;60(1)46-60.

2. Devarbhari PK, Vasudev MCR. Scorpion sting envenomation-an overview.J ClinBiomed Sci 2013;3(4):159-66.

3. Karnad DR. Management of scorpion envenomation:Need for a standard treatment protocol using drugs and antivenom. JAPI 2009;57(4):299-300.

4. Patil MM, Lakhkar B, Patil SV, Akki AS, Gobbur RH, Kalyanshettar SS. Scorpion sting envenomation, Vijayapur, Karnataka, India experience: new observations. IntJ ContempPediatr 201628;3(2):51823.

5. ChippauxJP,Goyffon M. Epidemiology of Scorpionism: a global appraisal. Acta Trop 2008;107(2):71-9. doi: 10.1016/j.actatropica.2008.05.021. Epub 2008 Jun 5.

6. Lakhkar BB, Patil MM, Patil SV. Scorpion Sting Envenomation in Children: A Literature Review.Indian J Trauma EmergPediatr2016;8(2):119-123.doi: http:// dx.doi.org/10.21088/ijtep.2348.9987.8216.14.

7. Joshi SR,Sapatnek SM.Stings and hopes: toxinomics and autonomic storm in Indian red
scorpion(Mesobuthustamulus, concanesis,pocock) JAPI 2007;55:11-3.

8. Bawaskar HS, Bawaskar PH.Utility of scorpion antivenom Vs Prazosin in the management of severe MesobuthusTamulus envenomation at rural setting. JAPI2007;55:14-21.

9. Mahadevan S. Scorpion sting.Indian Pediatr 2000(5)37:504-14.

10. Curry SC, VanceVV,Rayan PJ etal. Envenomation by scorpion centruroidssculpturatus.J ClinToxico/1983-84;21:417-49.

11. KhattabiA, Soulaymani-Bencheikh R, Achour S, Salmi LR. Classification of clinical consequences of scorpion stings: consensus development. Trans Roy Soc Trop Med Hyg 2011;105(7):364-69.

12. Bawaskar HS,Bawaskar PH.Prazosin therapy and scorpion envenoming.JAPI2000;48:1175-80.

13. Bawaskar HS, Bawaskar PH. Utility of scorpion antivenin vs prazosin in the management of severe Mesobuthustamulus (Indian red scorpion) envenoming at rural setting. JAPI 2007; 55:14-21.

14. Gupta V. Prazosin: a pharmacological antidote for scorpion envenomation. J Trop Pediatr 2006;52(2):15051. 